\title{
MODELO OLAM (OCEAN-LAND-ATMOSPHERE-MODEL): DESCRIÇÃO, APLICAÇÕES, E
} PERSPECTIVAS

\author{
RENATO RAMOS DA SILVA ${ }^{1}$, PEDRO LEITE SILVA DIAS ${ }^{2}$, DEMERVAL SOARES MOREIRA $^{3}$ E $^{-}$ \\ EVERALDO BARREIROS DE SOUZA ${ }^{4}$
}

\author{
${ }^{1}$ Universidade Federal da Bahia (UFBA) - Salvador-BA, Brasil \\ ${ }^{2}$ Laboratório Nacional de Computação Científica (LNCC) \\ ${ }^{3}$ Instituto Nacional de Pesquisas Espaciais (INPE) \\ ${ }^{4}$ Universidade Federal do Pará (UFPA)
}

renatosilva@ufba.br, pldsdias@lncc.br,demerval.moreira@cptec.inpe.br, everaldo@ufpa.br

Recebido Março 2008 - Aceito Abril 2009

\begin{abstract}
RESUMO
O modelo OLAM foi desenvolvido com objetivo de estender a capacidade de representar os fenômenos de escala global e regional simultaneamente. Este modelo apresenta inovações quanto aos processos dinâmicos, configuração de grade, estrutura de memória e técnicas de solução numérica das equações prognósticas. As equações de Navier-Stokes são resolvidas através da técnica de volumes finitos que conservam massa, momento e energia. No presente trabalho, apresenta-se uma descrição sucinta do OLAM e alguns resultados de sua aplicação em simulações climáticas da precipitação mensal para a região norte da América do Sul, bem como em rodadas para previsão numérica de tempo regional. Os resultados mostram que o modelo consegue representar bem os aspectos meteorológicos de grande escala. Em geral, seu desempenho melhora quando são adotadas grades de maior resolução espacial, nas quais se verificam melhorias significativas tanto na estimativa da precipitação mensal regional, quanto na previsão numérica de tempo.
\end{abstract}

Palavras Chave: OLAM, modelagem climática, previsão numérica de tempo

\begin{abstract}
OCEAN-LAND-ATMOSPHEREMODEL(OLAM):DESCRIPTION,APPLICATIONS, AND PERSPECTIVES.

The OLAM model was developed to extend the capability to represent the global and regional scale phenomena simultaneously. The model presents innovations regarding to the dynamic processes, grid configuration, memory structure, and numerical technique solutions for the prognostics equations. The Navier-Stokes equations are solved using the finite volume technique that conserves mass, momentum, and energy. In this study the OLAM model is described, and results are presented for its application on the climate mode to simulate the monthly prediction of precipitation for South America and numerical weather prediction. The results show that the model is able to represent reasonable the large scale meteorological processes. In general, its performance improves when grids of greater resolution is adopted, for which significant improvements are observed for the monthly precipitation simulation estimates as for the numerical weather prediction.
\end{abstract}

KeyWorks: OLAM, climate modeling, numerical weather prediction 


\section{INTRODUÇÃO}

No contexto atual das discussões de mudanças climáticas e desenvolvimento das previsões de cenários futuros de clima global e regional, tanto os estudos observacionais como a modelagem numérica são cruciais para o entendimento e geração de informações úteis a comunidade global. Estudos recentes têm evidenciado significativas mudanças climáticas globais nas últimas décadas com efeitos regionais bastante variáveis. Há significativo consenso na comunidade científica de que tais mudanças são resultantes de ações antropogênicas, principalmente através do aumento na concentração de gases causadores do efeito estufa (Cubash et al., 2001; Solomon et al., 2007). A mais notável mudança observada corresponde a um consistente aumento da temperatura global, que atingiu aproximadamente $0,8^{\circ} \mathrm{C}$ nos últimos cem anos (Solomon et al., 2007). Existem ainda outras evidências de alteração climática global. Novas observações mostraram que a área coberta por gelo marinho na região Ártica tem diminuído aproximadamente 7\% por década desde os anos 1970 (Stroeve et al., 2005). Recentes análises mostram também, que o número de furacões de maior intensidade (categorias 4 e 5 na escala Simpson) aumentou nas últimas décadas e que esses aumentos aparentemente estão relacionados com o aumento da temperatura dos oceanos (Emanuel, 2005; Webster et al., 2005).

Os impactos destas mudanças globais na América do Sul e particularmente na Amazônia ainda são incertos. No entanto, vários estudos mostram importantes relações entre a condição climática na América do Sul e o estado do clima em outras regiões. Por exemplo, sabe-se que temperaturas mais quentes do Atlântico Norte mantêm a Zona de Convergência Intertropical (ZCIT) em uma posição mais ao norte causando períodos de seca na região nordeste do Brasil e leste da Amazônia (Nobre e Shukla, 1996; Souza et al., 2000; Marengo, 2004; Marengo, 2005; Souza et al., 2005). Durante períodos de El Niño (La Niña), quando a Temperatura da Superfície do Mar (TSM) do Oceano Pacífico oriental aumenta (diminui) anormalmente, também se notam regimes de seca (enchentes) no nordeste do Brasil e intensas chuvas na região sudeste da América do Sul (Ropelewski e Halpert, 1987; Marengo e Hastenrath, 1993, Souza e Ambrizzi, 2002; Souza et al., 2004). Outras observações do nível dos rios da região sudeste e sul da América do Sul (i.e. Rios Negro, Paraguai, Paraná e Uruguai) mostram também uma forte relação de sua variabilidade com oscilações da TSM do Atlântico Norte (Robertson e Mechoso, 1998). Além do mais, estudos numéricos mostram uma importante relação entre a liberação de calor latente proveniente da forte convecção na região Amazônica e condições climáticas no Atlântico Norte e a Zona de Convergência do Atlântico Sul [ZCAS] (Grimm e Silva Dias, 1995). Observa-se, portanto, um importante controle dos fenômenos de grande escala no padrão de formação e evolução das ZCAS.

Existe ainda uma importante conexão entre o comportamento da ZCAS e o norte da América do Sul. O transporte de umidade oriundo da região amazônica que alimenta as ZCAS é o mais expressivo do Hemisfério Sul (Seluchi e Marengo, 2000). Este transporte se dá através de um intenso jato de baixos níveis localizado a leste dos Andes; jatos mais intensos geralmente estão associados com ZCAS mais intensas (Marengo et al., 2004; Wang e Fu, 2004).

Outros distúrbios remotos são fundamentais na determinação dos regimes climáticos. As oscilações MaddenJulian, por exemplo, possuem uma periodicidade da ordem de 30-60 dias, que influencia o regime climático na região tropical da América do Sul (Souza e Ambrizzi, 2006).

Além dos fenômenos e mecanismos de grande escala, vários processos meteorológicos locais possuem importante influencia no clima regional. Mais especificamente, no caso da Amazônia, temos o efeito das linhas de instabilidade (Greco et al., 1990; Garstang e Massie Jr, 1994; Cohen et al., 1995), circulações associadas à brisa fluvial (Silva Dias et al., 2004) e complexos convectivos que se desenvolvem localmente (Silva Dias et al. 2002a, 2002b; Ramos da Silva e Avissar, 2006).

Nos últimos anos vários fenômenos meteorológicos $\mathrm{e}$ climáticos têm sido observados na América do Sul, que podem estar relacionados com as alterações recentes do clima global. Por exemplo, um forte período de estiagem na região sudeste do Brasil, no ano de 2001, causou um extremo rebaixamento do nível dos rios e ocasionou em drástica diminuição na produção de energia hidroelétrica. Em 2004 observou-se o primeiro furacão a atingir a América do Sul (estado de Santa Catarina) de que se tem notícia (Ramos da Silva et al., 2004; Pezza e Simmonds, 2005). Em 2005 observou-se na Amazônia a seca mais severa dos últimos 40 anos (Marengo et. al., 2008).

Atualmente estudos do impacto de mudanças climáticas globais têm sido obtidos através de MCGs (Cubasch et al., 2001; Solomon et al., 2007; Marengo, 2007). Por exemplo, Cox et al. (2004) mostraram que o aumento esperado na concentração de $\mathrm{CO}_{2}$ para o século 21, deve produzir um padrão climático típico de El Niño permanente e causar aumento de temperatura e diminuição da precipitação na bacia Amazônica. Tais modelos, no entanto, têm resolução espacial da ordem de 2-5 graus de latitude-longitude e, portanto não conseguem simular bem as mudanças climáticas regionais. Por outro lado, modelos regionais (i.e. de área limitada) têm melhor resolução, mas precisam ser alimentados com condições atmosféricas de grande escala e, portanto não interagem com a escala global (Ramos da Silva et al., 2008). Até o presente momento não havia um modelo capaz de representar simultaneamente fenômenos meteorológicos de escala global e regional. Como solução para 
este impasse desenvolveu-se recentemente na Universidade Duke - EUA, um novo modelo que representa uma nova geração de modelos meteorológicos. Este modelo denominado "Ocean-Land Atmosphere Model" (OLAM) possui como principal característica a capacidade de representar fenômenos meteorológicos de escala global, e com o acoplamento de grades refinadas consegue representar de forma mais acurada os fenômenos de escala local e estimar o clima regional (Walko e Avissar, 2008).

A estrutura computacional e modelagem física do modelo OLAM são fundamentadas no "Regional Atmospheric Modeling System" (RAMS) cuja aplicação tem sido importante no entendimento e previsão de tempo e clima nos grandes centros de estudo meteorológico do Brasil. O modelo RAMS tem sido usado com sucesso em previsão numérica de tempo para a região nordeste (Ramos da Silva et al., 1996a; Ramos da Silva et al., 1996b) e sudeste do Brasil (disponível em http://www.master. iag.usp.br), e no monitoramento das emissões de aerossóis causadas por queimadas (Freitas, 1999) e poluentes na grande São Paulo (Freitas et al., 2005). Este modelo tem sido usado em vários estudos de impacto ambiental, como no entendimento dos efeitos causados pelo desmatamento da Amazônia no regime de chuvas (Ramos da Silva et al., 2002; Silva Dias et al., 2002a; Ramos da Silva e Avissar, 2003; Gandu et al., 2004; Ramos da Silva et al., 2008). O modelo foi usado também com sucesso na simulação e entendimento das características do furacão Catarina, e do impacto da TSM do Atlântico na sua evolução (Ramos da Silva et al., 2004). Desenvolveu-se, portanto um importante entendimento no uso do modelo RAMS nos centros de pesquisa meteorológica e climática do Brasil.

Baseado nos estudos de modelagem numérica de tempo e clima supracitados pode-se dizer que, para simular mais

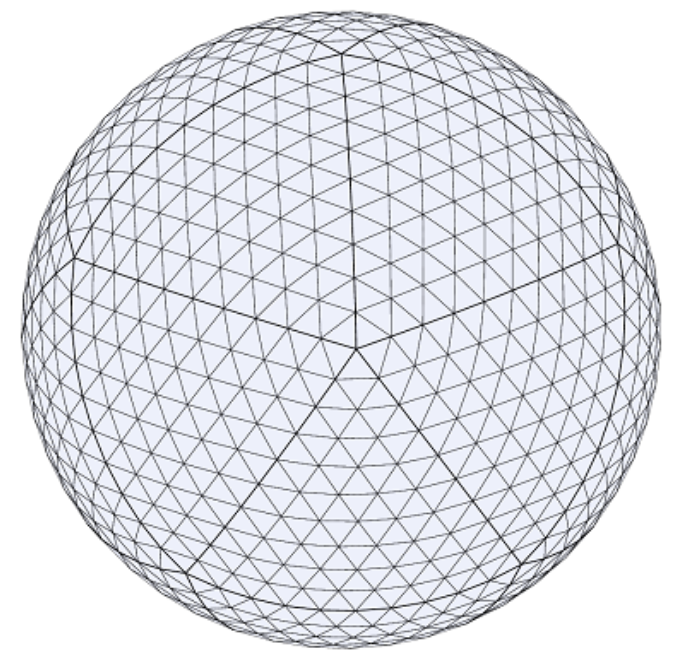

Figura 1 - Grade icosaédrica usada no modelo OLAM. Adaptada de Walko e Avissar (2008). realisticamente o regime de chuvas na Amazônia é necessário representar os mecanismos de grande escala, tais como as ondas de Rossby que se propagam sobre o Pacífico e o impacto da convecção anômala associada à variabilidade da TSM do Atlântico e Pacífico. Ao mesmo tempo é necessário representar com boa resolução os aspectos locais, como a reciclagem hidrometeorológica na Amazônia, os jatos de baixos níveis, topografia e cobertura superficial. Tais fenômenos de mesoescala são de grande importância na produção e distribuição de precipitação na Amazônia e na região de atuação da ZCAS. No presente momento, o único modelo capaz de simular a mútua interação entre fenômenos meteorológicos de várias escalas é o modelo OLAM.

Estudos preliminares com o modelo para alguns casos mostraram boa simulação dos principais centros convectivos da América do Sul (Ramos da Silva, 2006; Ramos da Silva et al., 2007). No presente trabalho, inicialmente é feita uma descrição sucinta das características do modelo, e a seguir são apresentadas as opções de configuração usadas nos testes para simulações climáticas sazonais e de previsão de tempo para a região da América do Sul. Posteriormente são apresentados os resultados obtidos, seguindo com uma discussão para futuras aplicações com o modelo.

\section{DESCRIÇÃO DO MODELO}

\subsection{Estrutura de grade}

O modelo OLAM usa uma grade do tipo não estruturada, em que as células possuem um formato triangular na direção horizontal e, portanto, formam um prisma pentaedral. Esta estrutura de células equilaterais permite preencher totalmente uma esfera, sendo conveniente para a representação da atmosfera terrestre. A triangulação da esfera terrestre se dá através de sua divisão em 20 triângulos eqüiláteros formando um icosaedro. Cada um destes triângulos originais é subdividido em outros triângulos, permitindo assim, melhoras na resolução espacial (Figura 1). As localizações das grades triangulares não possuem uma distribuição horizontal estruturada. Esta configuração (não-estruturada) requer uma definição da posição destas células através de índices de localização, que são armazenados computacionalmente e posteriormente usados durante a integração numérica. Na direção vertical, a célula pentaedral é construída através da projeção radial das superfícies da grade triangular a partir do centro da Terra. Desta forma, as faces verticais ficam alinhadas com a força de gravidade, e a área das faces horizontais se expandem gradualmente com a altura. A distância vertical entre estas faces pode ser ajustada de acordo com a aplicação de interesse. Geralmente, usa-se menor espaçamento próximo à superfície, onde ocorrem 
importantes processos relacionados à dinâmica da camada limite planetária, e maior espaçamento em níveis superiores. Sendo global, esta grade evita a necessidade de assimilação de dados meteorológicos durante a integração numérica como ocorre nos modelos de área limitada, embora seja também possível assimilar dados meteorológicos de um modelo global previamente rodado.

O acoplamento dos campos de massa e momento se dá através de uma grade do tipo Arakawa-C conforme descrito por Wenneker (2002). Este tipo de estrutura é um dos mais eficientes computacionalmente, e são convenientes para representação de fenômenos atmosféricos em amplo espectro de escalas. $\mathrm{Na}$ presente configuração, os campos de momento são calculados em cada face vertical da célula, e os campos escalares (pressão atmosférica, temperatura, etc) são estimados pelo seu valor médio volumétrico, e representado no centro do volume desta célula. Esta configuração de grade separa o cálculo de momento em uma componente vertical e outra horizontal. Outros tipos de modelo possuem estrutura vertical dependentes de projeções das componentes verticais e horizontais no cálculo do momento e, portanto, são menos eficientes computacionalmente.

Alta resolução espacial, para alguma região de interesse, pode ser obtida através da divisão das grades triangulares. Este método mantém na mesma localidade as faces do triângulo original da grade de menor resolução. Nas regiões internas, cada grade triangular equilateral é dividida em quatro novos triângulos (Figura 2). Este método evita sobreposição de grades de diferente resolução, portanto não necessita de algoritmos especiais de aninhamento, evita ruídos numéricos, e mantém a conservação de energia. Esta técnica de refinamento permite ilimitado aumento da resolução espacial, incluindo

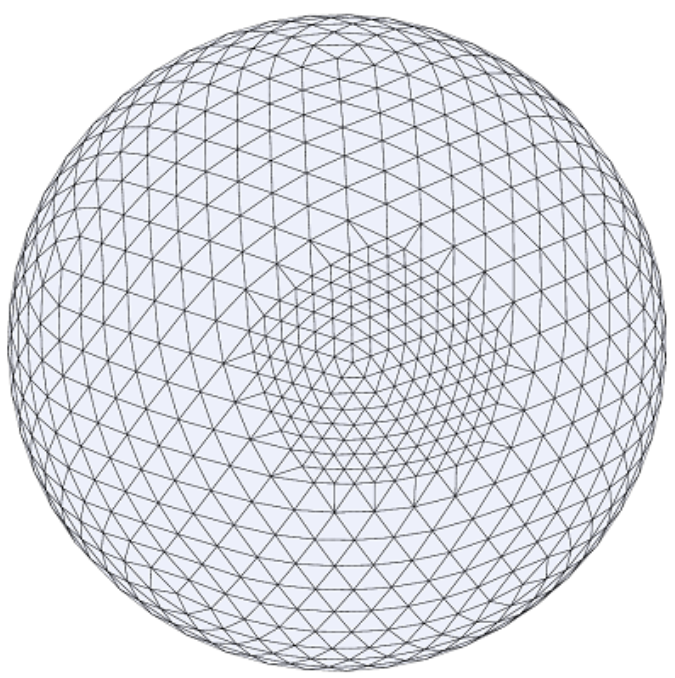

Figura 2 - Ilustração do refinamento das grades horizontais. Adaptada de Walko e Avissar, (2008). a possibilidade de inserir simultaneamente grades de alta resolução em regiões distintas.

O modelo OLAM representa a topografia por um método que assume grades superficiais fracionadas de seu volume original (Adcroft et al., 1997; Marshall et al., 1997). Este método mantém as superfícies de grade na horizontal, evitando os problemas existentes em outros métodos menos eficientes, em que as grades se ajustam ao terreno e necessitam de projeções especiais de termos verticais e horizontais (Messinger et al., 1988). Esta melhoria traz importantes benefícios na representação dos processos atmosféricos em regiões de topografia íngreme, como no caso da região dos Andes, onde geralmente ocorrem problemas de instabilidade numérica.

\subsection{Equações dinâmicas governantes}

O OLAM considera as leis de conservação de massa, momento e energia interna. Mais especificamente, são considerados a equação de conservação de momento nas direções horizontal e vertical, continuidade de massa, conservação de energia, conservação de outras variáveis escalares, e equação de estado. Por exemplo, a componente de conservação de momento na direção xi pode ser representada como:

$$
\begin{gathered}
\frac{\partial v_{i}}{\partial t}=-\nabla \cdot\left(v_{i} \vec{V}\right)-(\nabla p)_{i} \\
-(2 \rho \vec{\Omega} \times \vec{v})_{i}+\rho g_{i}+F_{i}
\end{gathered}
$$

em que, $\mathrm{V}_{\mathrm{i}}$ representa o tensor das componentes de vento, $p$ a pressão atmosférica, $\rho$ a densidade do $\operatorname{ar}, \Omega$ a velocidade angular da Terra, g a força gravitacional, e $F_{i}$ o tensor das forças de atrito.

\subsection{Discretização Numérica}

O OLAM resolve as equações de Navier-Stokes através do método de volumes finitos. A discretização espacial se dá através da integração sobre volumes de controle $(\psi)$ contornados por superfícies de área $(\sigma)$, conforme descrito em Adcroft et al. (1997) e Wenneker et al. (2002). Para os termos que envolvem divergência, o teorema de Gauss é aplicado para transformar as integrais de volume em integrais de superfície.

$$
\int_{\Psi} \nabla \cdot \vec{\phi} d \psi=\oint_{\sigma} \vec{\phi} \cdot d \sigma
$$

Cada ponto de grade comunica-se diretamente com os pontos de grade vizinhos.

A equação integral resultante para a conservação de momento na direção $\mathrm{x}_{\mathrm{i}}$ torna-se: 


$$
\begin{aligned}
& \frac{\partial}{\partial t} \int V_{i} d \psi=-\oint\left(v_{i} \vec{V}\right) \cdot d \vec{\sigma}-\int \frac{\partial p}{\partial x_{i}} d \psi \\
& -\int(2 \rho \vec{\Omega} \times \vec{v})_{i} d \psi+\int \rho g_{i} d \psi+\int F_{i} d \psi
\end{aligned}
$$

Os fluxos calculados em cada uma das faces do elemento de volume é dividido em contribuições do valor médio e de sub-grade. Todo o fluxo que passa por cada uma das faces é transportado para uma célula adjacente, mantendo desta forma a conservação de massa e energia.

Para a integração temporal, o modelo usa um passo de tempo longo para o cálculo da maioria dos termos prognósticos, e usa um passo de tempo menor para os termos acústicos como, por exemplo, para a força de gradiente de pressão. O esquema numérico de Adams-Bashforth de segunda ordem é usado na estimativa da maioria dos termos advectivos (Marshall et al., 1997).

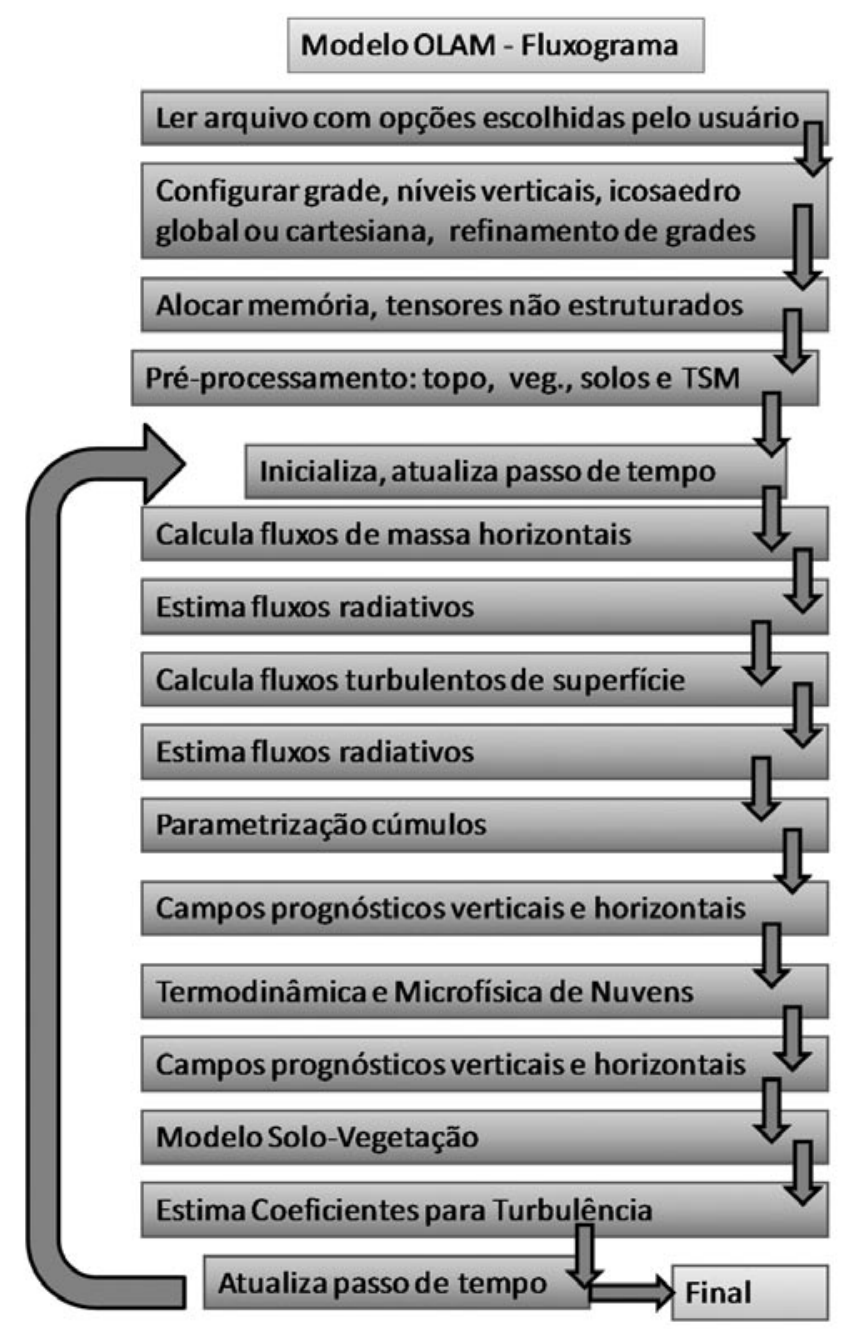

Figura 3 - Ilustração dos vários passos conduzidos durante uma integração numérica com o modelo OLAM.

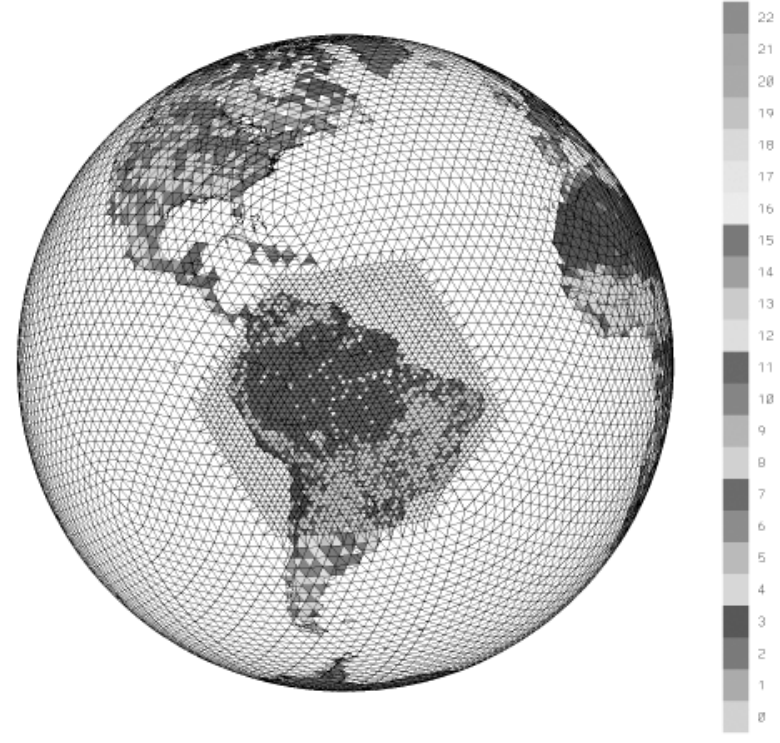

Figura 4 - Modelo OLAM, cobertura vegetal e domínio das grades global e grade acoplada para a América do Sul.

\subsection{Parametrizações Físicas}

As parametrizações físicas adotadas no OLAM são em sua grande maioria advindas do modelo de mesoescala RAMS (Pielke et al., 1992, Cotton et al., 2003). O modelo considera parametrizações físicas para difusão turbulenta (Mellor e Yamada, 1974), transferências de radiação de onda curta e onda longa (Chen e Cotton, 1987), formação de nuvens e precipitação em suas várias formas liquidas e sólidas (Meyers et al., 1997; Walko et al., 1995), processos convectivos associados a nuvens cumulus (Tremback, 1990), e as trocas de calor sensível e latente entre a superfície e a atmosfera, incluindo várias camadas de solo, e um modelo para o dossel da vegetação (Avissar e Pielke, 1989; Walko et al., 2000).

A Figura 3 apresenta um fluxograma dos vários passos conduzidos pelo modelo durante a integração numérica. Inicialmente, várias tarefas são executadas na fase de préprocessamento, como configuração de grade, e inicialização das condições de contorno. A seguir, inicia-se a integração dos processos físicos e dinâmicos, que são atualizados a cada passo de tempo até chegar ao final da integração.

\section{METODOLOGIA, CONFIGURAÇÃO DO MODELO E APLICAÇÕES}

A seguir são descritos as configurações usadas nos experimentos numéricos, suas aplicações para previsão climática e de tempo, e os dados observacionais usados na avaliação do modelo. 


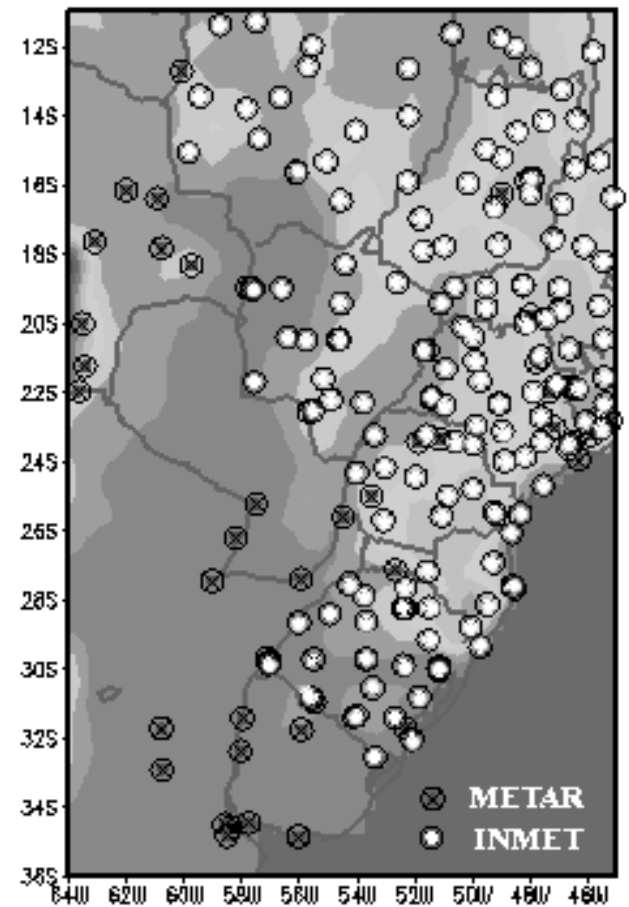

Figura 5 - Localizações das estações usadas nos testes para previsão numérica de tempo. Obtido do sistema MASTER (disponível em www. master.iag.usp.br).

\subsection{Descrição dos Experimentos Numéricos de Previsão Climática Sazonal}

Com objetivo de avaliar o desempenho do modelo na previsão climática mensal, vários testes foram feitos e seus resultados foram comparados com dados de precipitação acumulada mensal. Inicialmente, o modelo foi configurado com uma grade global e outras grades mais refinadas foram adicionadas para a região da América do Sul. A Figura 4 ilustra a localização de uma grade mais refinada inserida no domínio global e as respectivas características de superfície. Com o objetivo de avaliar o efeito da resolução espacial, testes foram feitos usando duas, três e quatro grades. Inicialmente, o modelo foi configurado com duas grades, a grade global com células espaciais de aproximadamente $200 \mathrm{~km}$ e outra grade refinada com aproximadamente $100 \mathrm{~km}$. Posteriormente, testes com três grades foram executados com espaçamento horizontal de 200 $\mathrm{km}, 100 \mathrm{~km}$ e $50 \mathrm{~km}$, respectivamente. Finalmente, novos testes configurados com quatro grades, possibilitaram um espaçamento de $25 \mathrm{~km}$ para a grade mais refinada. O modelo assimila condições de contorno superficiais de topografia, vegetação e textura de solo. Vários testes foram executados para os períodos entre junho e dezembro de 2007, permitindo avaliar o modelo em períodos de clima seco, de transição e chuvoso. O modelo foi inicializado com dados atmosféricos globais obtidos do NCEP, e para a Temperatura da Superfície do Mar (TSM) foi assumida a média climatológica do respectivo mês de estudo. Integrações numéricas foram executadas em vários testes que tiveram tempo total de 30, e 90 dias. Sendo o OLAM um modelo configurado com grade global, condições meteorológicas de fronteiras não foram necessárias.
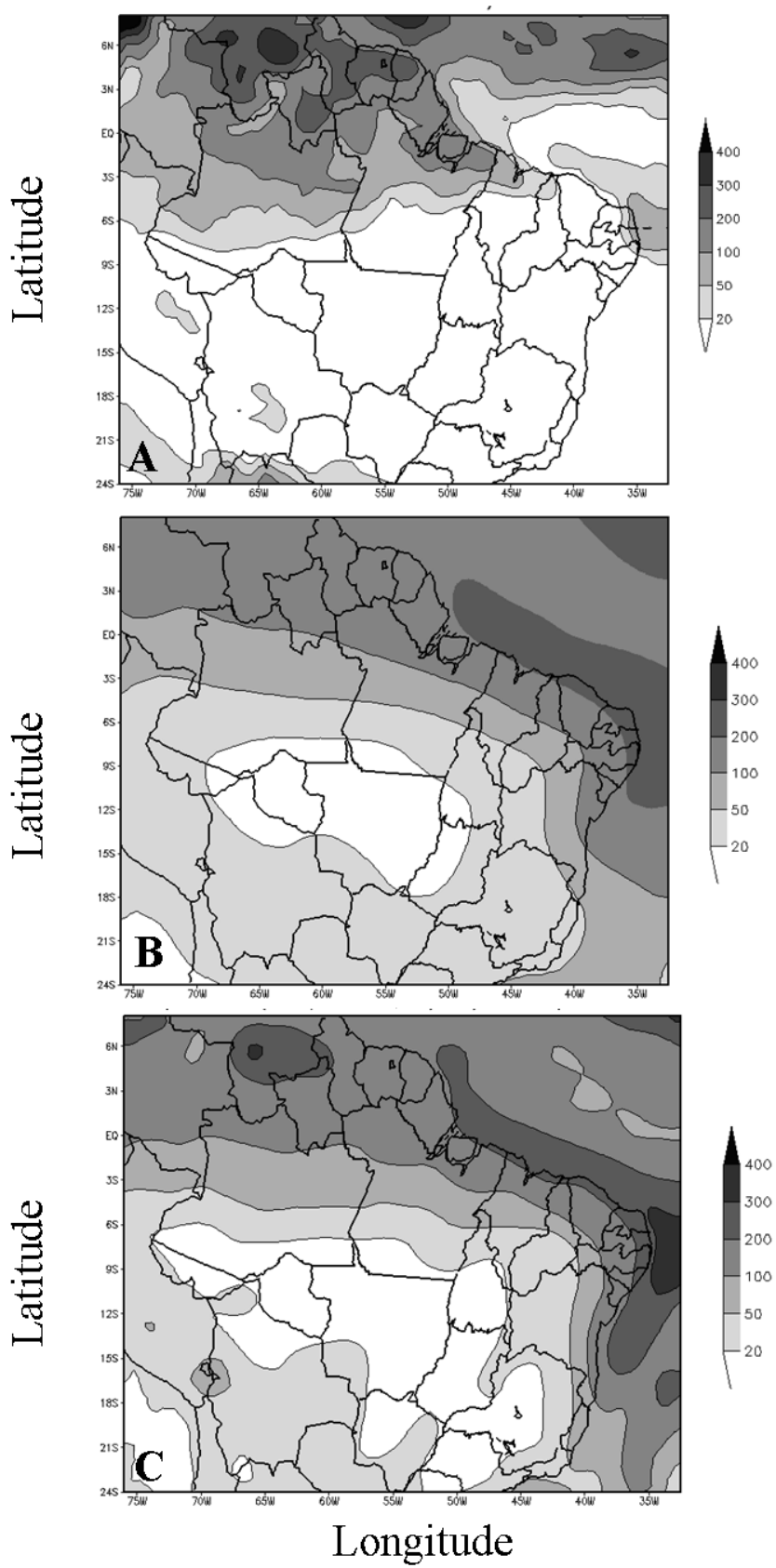

Figura 6 - Precipitação acumulada para junho de 2007, a) observada (Fonte: GPI), b) simulada pelo OLAM com duas grades $(200 \mathrm{~km} \mathrm{e}$ $100 \mathrm{~km})$, e c) três grades $(200 \mathrm{~km}, 100 \mathrm{~km}, 50 \mathrm{~km})$. 


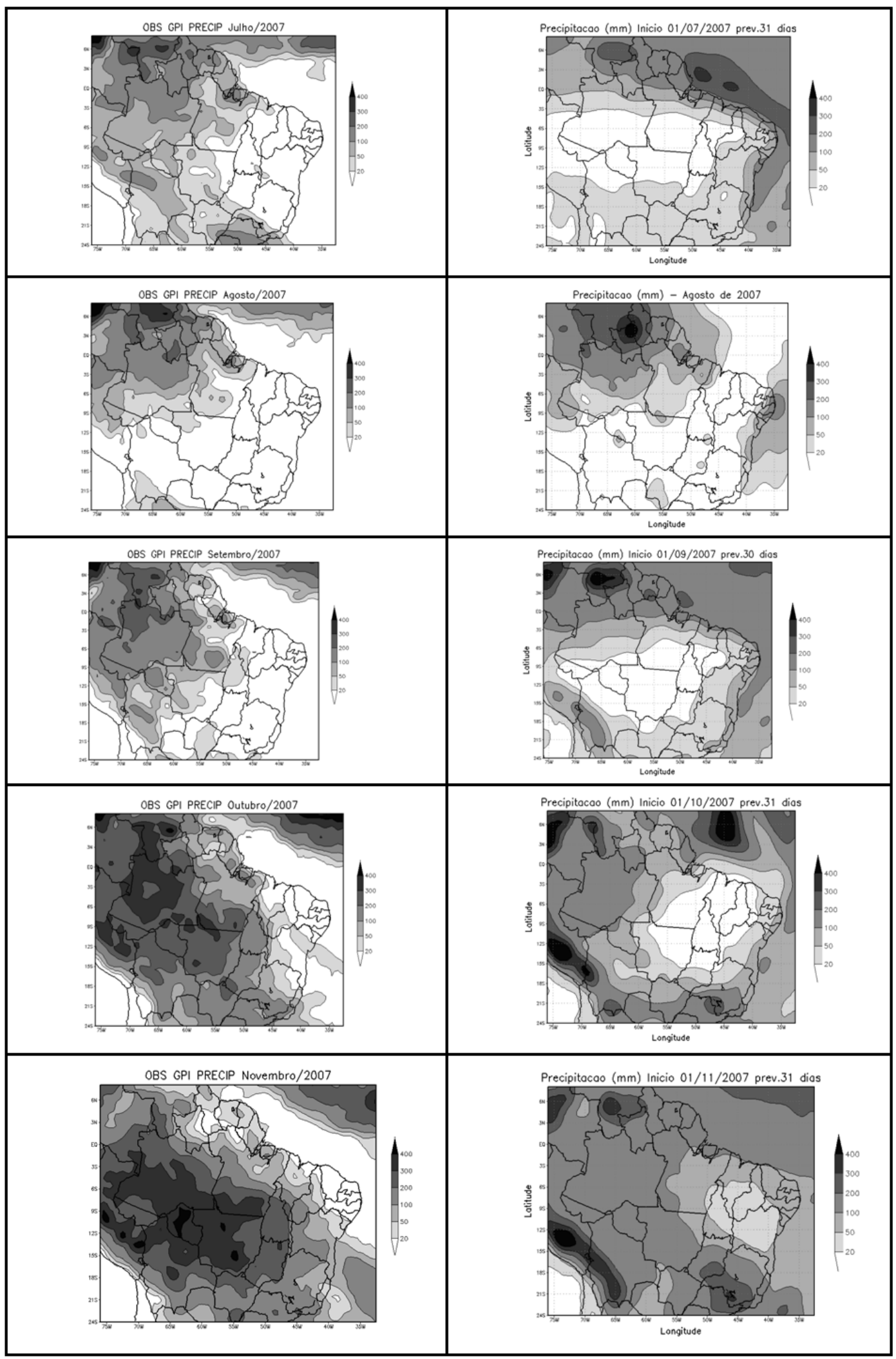

Figura 7 - Precipitação acumulada observada GPI (esquerda), e simulada pelo OLAM (direita). 


\subsection{Descrição dos Experimentos em Previsão Numérica de Tempo}

O modelo OLAM tem sido rodado diariamente no CPTEC-INPE para avaliar seu desempenho na previsão numérica de tempo. Nestes testes preliminares, o modelo possui três tipos de configurações: $i$ ) resolução da ordem de $250 \mathrm{~km}$ com a microfísica de nível 3, que considera os hidrometeoros de nuvens (não apresentado); ii) resolução da ordem de $250 \mathrm{~km}$ com microfísica de nível 1 que representa uma atmosfera seca (OLAMCg1); e iii) resolução da segunda grade da ordem de 125 $\mathrm{km}$ com microfísica de nível 1 (OLAMNg2). O modelo roda a partir de condições iniciais das análises globais do NCEP por um tempo total de 72 horas.

\subsection{Descrição dos Dados Meteorológicos Observados}

Para avaliação das simulações de 30 e 90 dias, dados de precipitação mensal acumulada foram obtidos do NCEP, disponíveis em ftp.cpc.ncep.noaa.gov/precip. Duas fontes de dados foram usadas, a precipitação em pontos de grade $1^{\circ} \mathrm{x}$ $1^{\circ}$ interpolada a partir de dados das estações pluviométricas (NCEP), e também a precipitação estimada por satélite (GPIGoes Precipitation Index), cuja resolução espacial é de $1^{\circ} \times 1^{\circ}$. Detalhes sobre os dados de precipitação do NCEP, podem ser encontrados em Silva et al. (2007) e sobre o GPI em Arkin e Meisner (1987).
Para avaliação das rodadas diárias do CPTEC-INPE, o modelo foi comparado com dados de temperatura observados das estações dos aeroportos (METAR) e das estações telemétricas do INMET, e também com resultados do modelo de previsão de tempo global do CPTEC e do NCEP (Moreira et al., 2006). A Figura 5 ilustra a localização das estações usadas nestas avaliações.

\section{RESULTADOS}

Nas seções seguintes são apresentados resultados preliminares do uso do modelo OLAM na simulação da precipitação mensal e da previsão numérica de tempo.

\subsection{Previsão Climática Sazonal}

Nestes testes o modelo foi rodado para meses entre junho e dezembro de 2007, correspondendo a períodos secos e úmidos. Vários testes foram executados para avaliar o desempenho do modelo em relação à sua configuração de grade, principalmente da resolução espacial.

A precipitação acumulada, observada no mês de junho de 2007, mostra pouca precipitação nas regiões central da América do Sul, e regiões de maior acumulação no norte da Amazônia e leste do Nordeste Brasileiro (Fig. 6a). Comparando com a simulação feita pelo OLAM para o mesmo período usando-se apenas duas grades (200 km e $100 \mathrm{~km}$ ), nota-se que o modelo

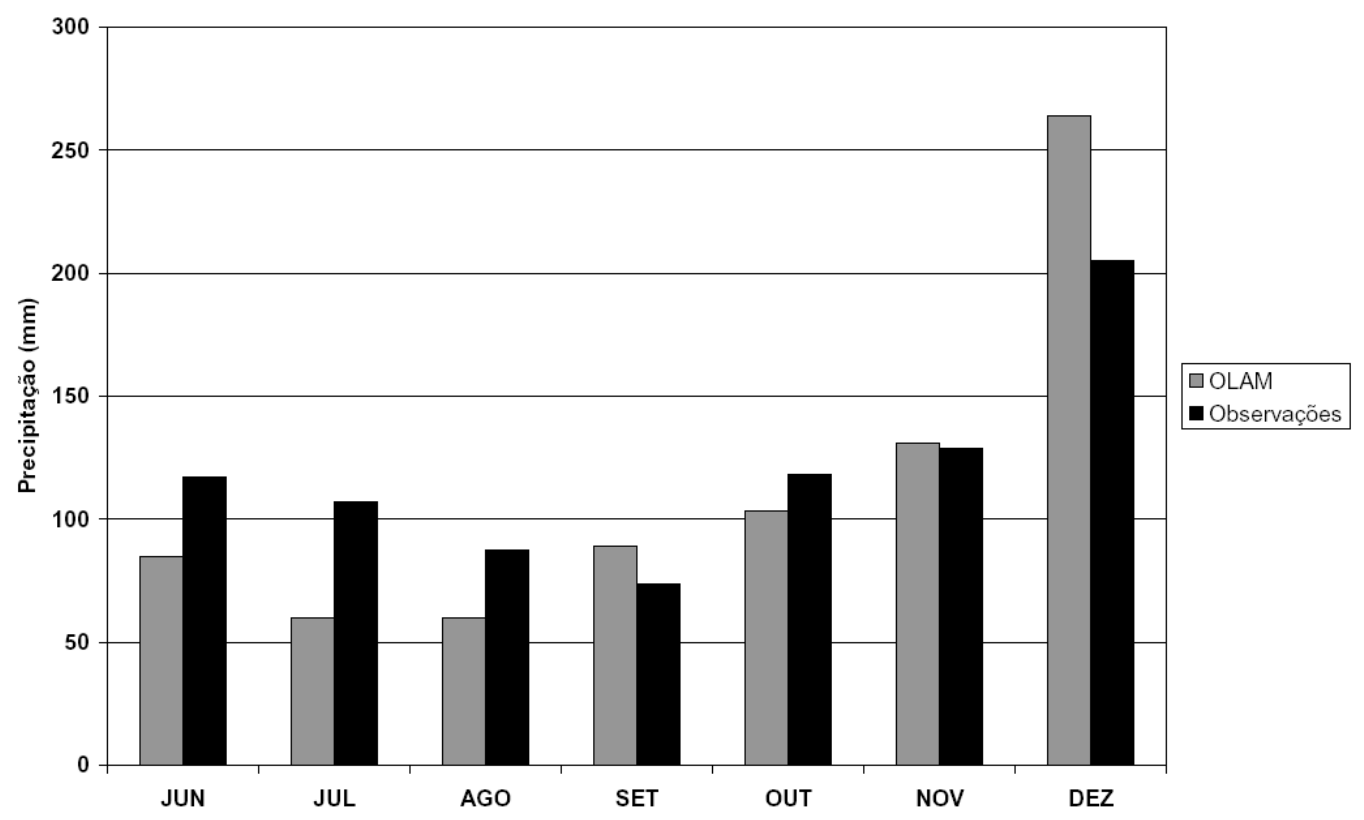

Figura 8 - Precipitação média acumulada para a área entre as latitudes 13S e $6 \mathrm{~N}$ e longitudes $75 \mathrm{~W}$ e $45 \mathrm{~W}$, simulada pelo OLAM e observada (NCEP). 
representa bem as áreas de estiagem, mas não simula muito bem os centros de maior precipitação (Fig. 6b). Nota-se, entretanto, que a inclusão de uma terceira grade, e, portanto uma melhoria da resolução espacial com células de grade de aproximadamente $50 \mathrm{~km}$, proporciona uma sensível melhora na representação das áreas de maior acumulação de chuva. Estas melhorias ocorrem principalmente nas regiões de topografia mais elevada como na divisa com a Venezuela, e no leste do nordeste brasileiro (Fig. 6c). Estes resultados levaram à adoção de três grades nos testes seguintes.

A Figura 7 apresenta mapas da precipitação mensal acumulada observada comparada com os resultados do modelo configurado com três grades para os meses de julho, agosto, setembro, outubro e novembro de 2007. As observações mostram que entre julho e setembro, houve períodos de estiagem principalmente nas regiões central do Brasil e sul da Amazônia. A acumulação obtida pelo modelo prevê com êxito esta situação e indica regiões de alta precipitação no noroeste da Amazônia em concordância com as observações. Nos meses de outubro e novembro ocorre um aumento da precipitação acumulada em várias regiões, principalmente no oeste e norte da Amazônia, e central e sudeste do Brasil. Este aumento ocorre principalmente devido à posição mais ao sul da ZCIT, e da atividade convectiva associada à ZCAS. Os resultados do modelo mostram um aumento da precipitação acumulada para estes meses, mas os centros de maior acumulação ocorrem nas encostas dos Andes e nas regiões montanhosas do norte. A deficiência da simulação da precipitação no oeste da Amazônia sugere que este deve ser um problema de resolução espacial, pois uma grade de $50 \mathrm{~km}$ não é capaz de simular bem as grandes linhas de instabilidade

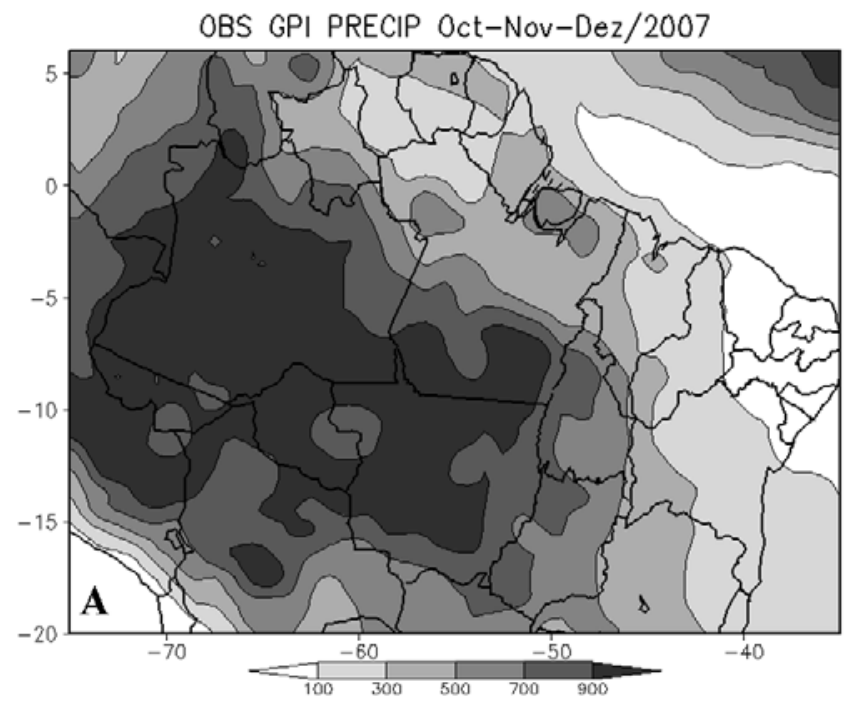

que são responsáveis pela maior parte da precipitação nesta região (Ramos-da-Silva et al., 2008). Embora a distribuição espacial da precipitação não seja bem representada, ocorre boa concordância entre a precipitação média espacial simulada e os valores observados (Fig. 8).

Existe atualmente grande interesse em previsão climática regional para períodos de três meses. Com este objetivo, o modelo foi integrado por 90 dias para o período entre outubro e dezembro de 2007 . Neste novo teste, o modelo foi configurado com quatro grades aumentando ainda mais a resolução espacial permitindo um espaçamento de grades da ordem de $25 \mathrm{~km}$. Desta forma, espera-se uma melhor representação da precipitação associada às grandes linhas de instabilidade. A Figura 9 apresenta resultados da precipitação acumulada obtidos com o modelo comparados com dados observados (GPI). Nota-se que com a melhor resolução, ocorre de fato uma melhor simulação da precipitação no oeste da Amazônia, no entanto, dados observados baseados em satélite sugerem que o modelo subestima a precipitação. Embora as estimativas baseadas em satélite sejam importantes por incluir áreas com ausência de dados observacionais, como os oceanos e as regiões montanhosas, existe uma aparente superestimação da precipitação acumulada para o período de estudo. De fato, enquanto a média espacial simulada acumulada foi de $497 \mathrm{~mm}$, as observações do NCEP baseadas em estações pluviométricas foram de $452 \mathrm{~mm}$ (ver fig. 8), e a estimativa baseada através do satélite (GPI) foi de $682 \mathrm{~mm}$.

A Figura 10 apresenta uma comparação entre a precipitação acumulada observada (GPI) e simulada pelo OLAM, para o período entre outubro e dezembro de 2007,

Figura 9 - Precipitação acumulada para outubro-novembro-dezembro 2007, (a) observada (GPI), e (b) simulada pelo modelo OLAM com 4 grades, com a de maior resolução tendo aproximadamente $25 \mathrm{~km}$ de espaçamento horizontal.

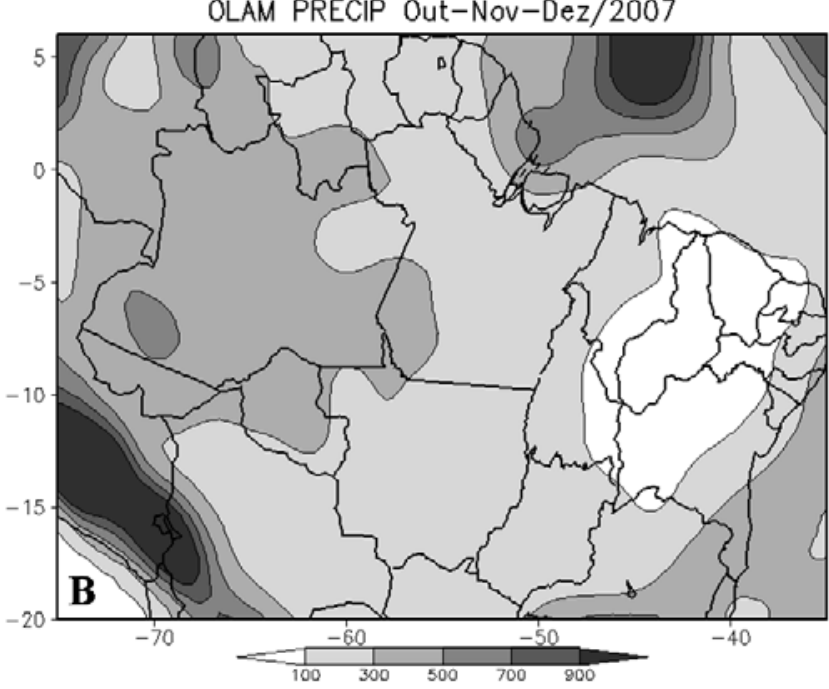


para todo o globo entre as latitudes $40 \mathrm{~S}$ e $40 \mathrm{~N}$. Estes resultados revelam que, embora o modelo represente bem a localização dos fenômenos de grande escala, estes fenômenos aparecem enfraquecidos e mais espalhados, muito provavelmente devido à baixa resolução da grade global (aprox. $200 \mathrm{~km}$ ). Devido à baixa resolução espacial, a ZCIT (Zona de Convergência InterTropical) e a ZCPS (Zona de Convergência do Pacífico Sul) aparecem enfraquecidas (Fig. 10b) e a precipitação associada acumula-se por uma área maior do que a observada (Fig. 10a). Ocorre também, subestimação da precipitação continental tropical como observadas nas regiões da América do Sul e África. A precipitação média acumulada para todo domínio de $250 \mathrm{~mm}$ é ligeiramente inferior à observada, que foi de 268 $\mathrm{mm}$, indicando possíveis problemas devidos à baixa resolução espacial. Outro possível problema pode estar relacionado com a parametrização de nuvens cumulus usada no modelo (Kuo, 1974). Estes resultados mostram que é muito desejável a grade global possuir melhor resolução espacial, e que testes com outras opções de parametrização cumulus devem ser realizadas. Algumas melhorias devem ocorrer com a nova versão do modelo, que vai permitir o uso de computação paralela de alto desempenho e, portanto melhor resolução espacial, e novas opções de parametrizações físicas como a parametrização de cumulus descrita por Grell (1993).

\subsection{Previsão Numérica de Tempo}

A Figura 11 foi obtida do sistema de avaliação do MASTER (Moreira et al., 2006) com algumas adaptações para melhor apresentação gráfica. Esta figura apresenta uma comparação entre a temperatura prevista pelo modelo em relação aos dados observados, e outros modelos de previsão de tempo para o mês de janeiro de 2008. Os resultados com o modelo OLAM são comparados com o modelo global do CPTEC com resolução de $100 \mathrm{~km}$ e o global do NCEP com resolução de $1^{\circ}$. Pode-se observar na Figura $11 \mathrm{a}$, que o erro quadrático médio do OLAMCg1 é significativamente maior que o do OLAMNg2, justificando assim a utilização da segunda grade para aumentar a resolução na região de interesse. Observa-se também, nas Figuras 11a e 11b, que o OLAMNg2 apresenta erros comparáveis ao global do NCEP (AVNg1) e do CPTEC (CPTECg1), mesmo com a melhor resolução da segunda grade do OLAM em relação a resolução destes modelos. Deve-se salientar, que a inclusão de uma nova grade demanda um custo computacional elevado, e por este motivo, a implementação do paralelismo no OLAM é de suma importância para torná-lo um modelo operacional de previsão de tempo.

\section{DISCUSSÃO E CONCLUSÕES}

O bom desempenho, obtido com o modelo OLAM na simulação dos fenômenos de escala local e global, mostra que o mesmo tem grande potencial de tornar-se uma ferramenta para previsão e avanço no entendimento dos processos meteorológicos e climáticos regionais. Os resultados mostram que existe uma necessidade de melhorias na resolução espacial, principalmente para melhorar a representação dos processos meteorológicos que afetam o clima regional, como as linhas de instabilidade.
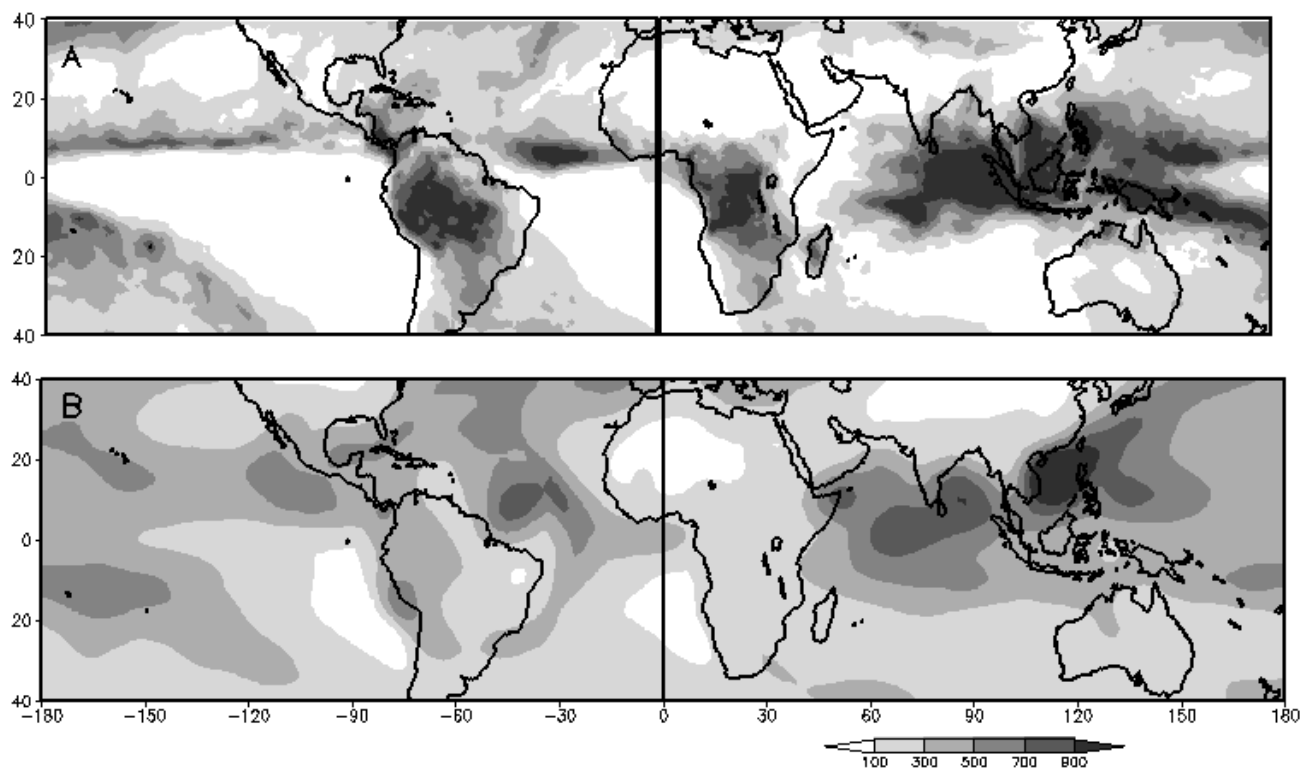

Figura 10 - Precipitação acumulada para outubro-novembro-dezembro 2007, (a) observada (GPI), e (b) simulada pelo modelo OLAM. 
Outras melhorias precisarão ser implementadas para se obter melhor desempenho do modelo. Entre estas se citam algumas que deverão ser conduzidas em breve como a paralelização do código fonte do modelo, para permitir simulações mais rápidas e melhorar a resolução; a adoção de várias opções de parametrizações físicas já usadas e testadas no modelo RAMS; a adoção de um modelo de vegetação dinâmica; e o acoplamento com um modelo oceânico.

A nova técnica de volumes finitos usada pelo OLAM permite ilimitada capacidade de refinamento de grade. A Figura12 ilustra um exemplo, em que o modelo foi configurado com 10 níveis de refinamento espacial para uma região próxima da Ilha do Marajó. Neste exemplo, a grade global foi configurada com um espaçamento da ordem de $200 \mathrm{~km}$ (Fig. 12a), e após o refinamento a grade de maior resolução chegou a um espaçamento de aproximadamente $400 \mathrm{~m}$ (Fig. 12b). Este exemplo mostra a possibilidade de simulação simultânea da interação entre processos meteorológicos de várias escalas.

Em geral este novo modelo propicia uma versatilidade importante devido à sua capacidade de representar simultaneamente os fenômenos locais como sistemas de brisa, os padrões regionais, e os processos globais representados por
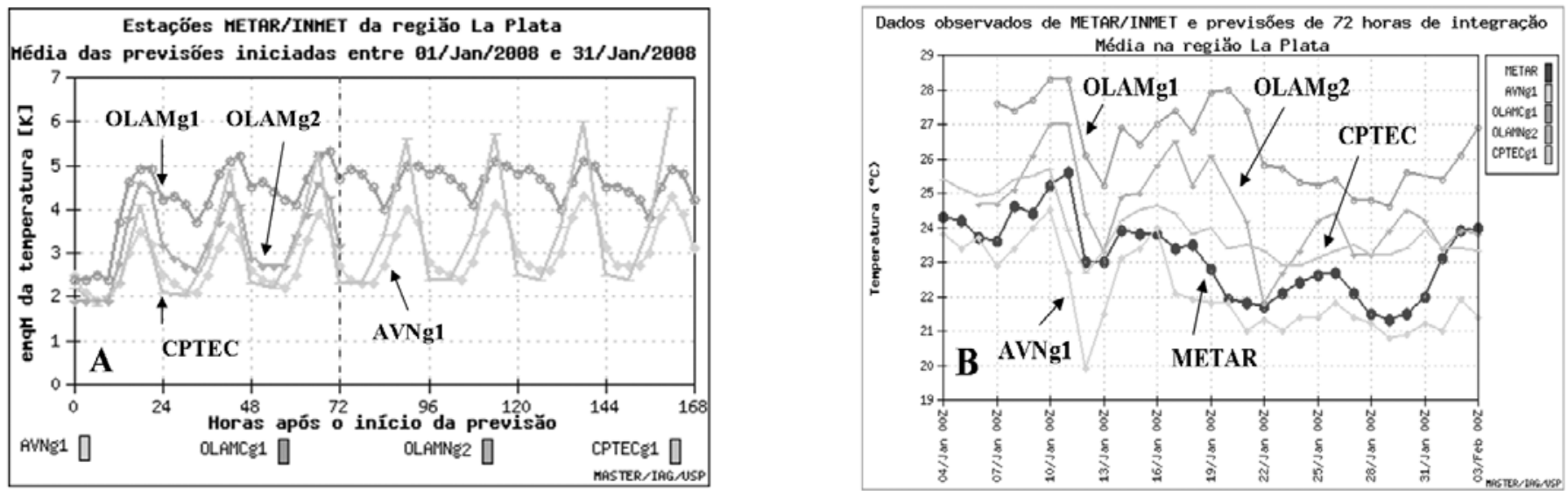

Figura 11 - Avaliação da temperatura média prevista: pelo OLAM operacional do CPTEC com resolução de $250 \mathrm{~km}(\mathrm{OLAMCg} 1)$ e $125 \mathrm{~km}$ (OLAMNg2); pelo global do CPTEC (CPTECg1) e pelo global do CNEP (AVNg1), no período de 01 a 31/jan/2008. (a) Erro quadrático médio ao longo de 168 horas de previsão e (b) Temperatura média das previsões de 72 horas de integração.

Adaptado de: http://www.master.iag.usp.br/intercomp/vies_emq.
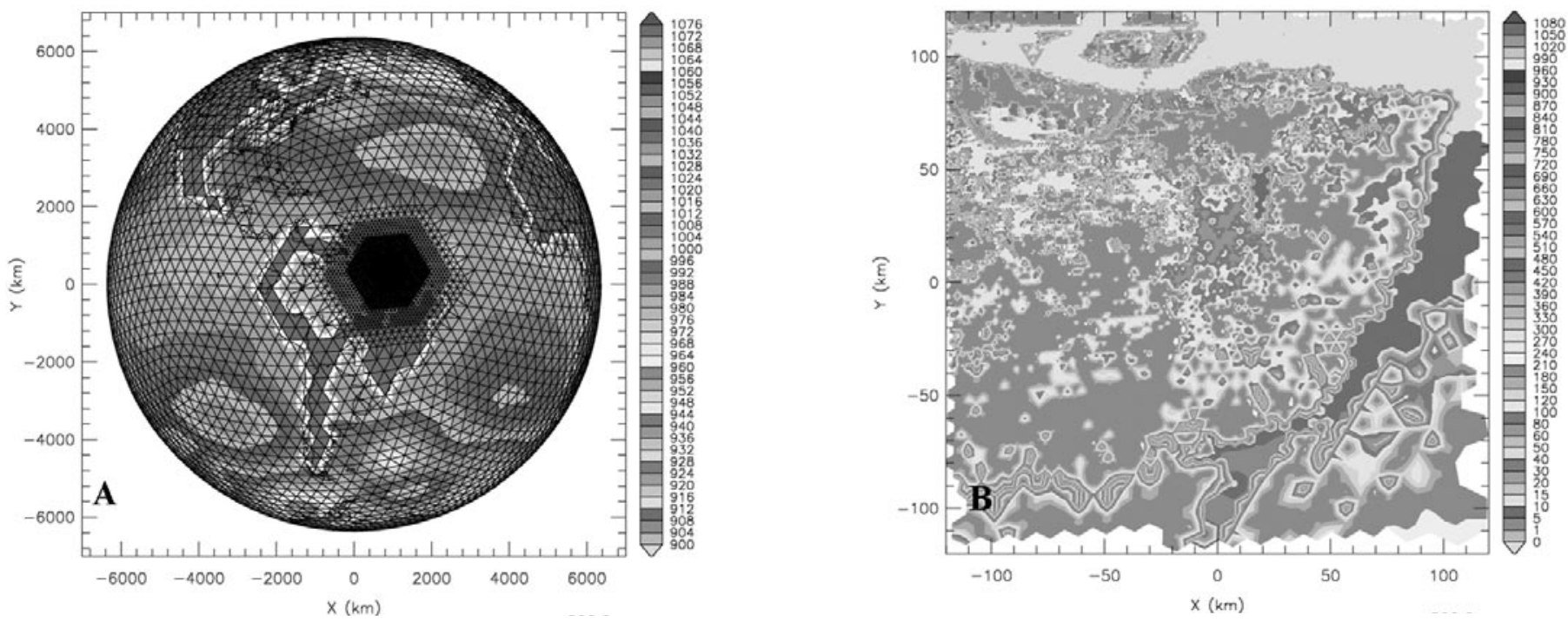

Figura 12 - Estrutura das grades usadas com o modelo OLAM para uma aplicação contendo 10 níveis de refinamento: (a) grade global com célula de aproximadamente $200 \mathrm{~km}$, e (b) grade de altíssima resolução localizada na região da Ilha de Marajó com espaçamento de aproximadamente $400 \mathrm{~m}$. 
teleconexões e propagação de ondas atmosféricas de larga escala. Citam-se como exemplos possíveis, efeitos da formação da brisa local no desenvolvimento das grandes linhas de instabilidade amazônicas. Adicionalmente, o modelo permite a interação entre fenômenos de escala local e de larga escala, podendo ser usado para o estudo de possíveis impactos do desmatamento local da Amazônia no clima tropical e global. O modelo OLAM deve produzir também, importantes avanços no entendimento das mudanças climáticas regionais causadas por fenômenos globais, cujos estudos até então só foram produzidos através de modelos globais de baixa resolução ou com modelos de área limitada.

\section{REFERÊNCIAS}

ADCROFT, A.; C. HILL; J. MARSHALL. Representation of topography by shaved cells in a height coordinate ocean model. Mon. Wea. Rev., 125, 2293-2315, 1997.

ARKIN, P.A.; B. MEISNER. The relationship between largescale convective rainfall and cold cloud over the Western Hemisphere during 1982-1984. Mon. Wea. Rev., 115, 5174, 1987.

AVISSAR, R.; R. PIELKE, A parameterization of heterogeneous land surfaces for atmospheric numerical-models and its impact on regional meteorology. Mon. Wea. Rev., 117, 2113-2136 1989.

BARREIRO, M., P.; CHANG,; R. SARAVANAN. Simulated precipitation response to SST forcing and potential predictability in the region of the South Atlantic convergence zone. Clim. Dyn., 24, 105-114, 2005.

CHEN, C.; W. COTTON. The physics of the marine stratocumulus-capped mixed layer. J. Atmos. Sci., 44, 2951-2977, 1987.

COHEN, J. C. P.; M. DIAS; C. A. NOBRE. Environmentalconditions associated with Amazonian squall lines - a casestudy. Mon. Wea. Rev., 123, 3163-3174, 1995.

COTTON W.R.; PIELKE R.A. SR;WALKO R.L.; LISTON G.E.; TREMBACK, C.J.; JIANG H.; MCANELLY R.L.; HARRINGTON J.Y.; NICHOLLS M.E.; CARRIO G.G.; MCFADDEN L.P. RAMS: Current Status and future directions. Meteorol. Atmos. Phys. 82: 5-29, 2003.

COX, P. M.; A. BETTS; M. COLLINS; P. P. HARRIS; C. HUNTINGFORD; C. JONES. Amazonian forest dieback under climate-carbon cycle projections for the 21st century. Theor. Appl. Climatol., 78, 137-156, 2004.

CUBASCH, U.; e COAUTHORS. Projections of future climate change. Climate Change 2001: The Scientific Basis, J. T. Houghton, Y. Ding, D. J. Griggs, M. Noguer, and P. J. van der Linden, Eds., Cambridge Univ. Press, 525-582, 2001.

EMANUEL, K. A. Increasing destructiveness of tropical cyclones over the past 30 years. Nature. 436, 686-688, 2005.

FREITAS, E. D.; L. D. MARTINS; P. L. SILVA DIAS; M. F. ANDRADE. A simple photochemical module implemented in RAMS for tropospheric ozone concentration forecast in the metropolitan area of Sao Paulo, Brazil: Coupling and validation. Atmos. Environ., 39, 6352-6361, 2005.

FREITAS, S. R. Numerical Modeling of the transport and emissions from biomass burning on South America tropical forest and savanna., Institute of Physics, University of Sao Paulo, 204 pp. [Available from Physics Dept., University of S.Paulo, S.Paulo-SP, Brazil.], 1999.

GANDU, A. W.; J. C. P. COHEN; J. R. S. SOUZA. Simulation of deforestation in eastern Amazonia using a high-resolution model. Theor. Appl. Climatol., 78, 123-135, 2004.

GARSTANG, M.; H. L. MASSIE J.R. Amazon squal lines: part I: Structure and kinematics. Mon. Wea. Rev. 122, 608622, 1994.

GRECO, S.; e COAUTHORS. Rainfall and surface kinematic conditions over Central Amazonia during ABLE 2B. J. Geophys. Res., 95, 17001-17014, 1990.

GRELL, G. A., Prognostic evaluation of assumptions used by cumulus parameterizations. Mon. Wea. Rev., 121, 764 787.

GRIMM, A. M.; P. L. SILVA DIAS. Analysis of tropical extratropical interactions with influence functions of a barotropic model. J. Atmos. Sci., 52, 3538-3555, 1995.

KUO, H. L., Further studies of the parameterization of the effect of cumulus convection on large scale flow. J. Atmos. Sci., 31, 1232-1240.

MARENGO, J. A.; S. HASTENRATH. Case-studies of extreme climatic events in the Amazon basin. J. Climate. 6, 617-627, 1993.

MARENGO, J. A. Interdecadal variability and trends of rainfall across the Amazon basin. Theor. App. Clim., 78, 79-96, 2004.

MARENGO, J. A.,; W. R. SOARES; C. SAULO; M. NICOLINI. Climatology of the low-level jet east of the Andes as derived from the NCEP-NCAR reanalyses: Characteristics and temporal variability. J. Climate. 17, 2261-2280, 2004.

MARENGO, J. Characteristics and variability of the water budget in the Amazon Basin. Clim. Dyn., 24, 11-22, 2005.

MARENGO, J. A., Mudanças Climáticas Globais e seus Efeitos sobre a Biodiversidade: caracterização do clima atual e definição das alterações climáticas para o território brasileiro ao longo do Sec. XXI . Série Biodiversidade (v.26), Ministério do Meio Ambiente, 212p, 2007.

MARENGO, J. A., Hydro-climatic and ecological behavior of the drought of Amazonia in 2005, Phil. Trans R. Soc. B., DOI 10.1098/rstb.2007.0026, 2008. 
MARSHALL, J.; A. ADCROFT; C. HILL; L. PERELMAN; C. HEISEY. A finite-volume incompressible Navier-Stokes Model for Studies of Ocean on Parallel Computers. J. Geophys. Res., 102, no. C3, 5753-5766, 1997.

MELLOR, G. L.; T. YAMADA. A hierarchy of turbulence closure models for planetary boundary layers. J. Atmos. Sci., 31, 1791-1806, 1974.

MESINGER, F.; Z. JANJIC; S. NICKOVIC; D. GAVRILOV; D.G. DEAVEN. The step-mountain coordinate: Model description and performance for cases of alpine lee cyclogenesis and for a case of an Appalachian redevelopment. Mon. Wea. Rev., 116, 1493-1520, 1988.

MEYERS, M. P.; R. L. WALKO; J. Y. HARRINGTON; W. R. COTTON. New RAMS cloud microphysics parameterization .2. The two-moment scheme. Atmos. Res., 45, 3-39, 1997.

MOREIRA, D. S.; SILVA DIAS, P. L.; LUCIO, P. S. Sistema de Avaliação Estatística de Modelos Numéricos de Previsão do Tempo. In: XIV Congresso Brasileiro de Meteorologia, 2006, Florianópolis. CDROM de trabalhos do congresso, 2006.

NOBRE, P.; J. SHUKLA. Variations of sea surface temperature, wind stress, and rainfall over the tropical Atlantic and South America. J. Clim., 9, 2464-2479, 1996.

PEZZA, A. B.; I. SIMMONDS. The first South Atlantic hurricane: unprecedent blocking, low shear and climate change. Geophys. Res. Lett., 32, doi:10.129/2005GL023390, 2005.

PIELKE, R. A. e CO-AUTORES. A comprehensive meteorological modeling system - RAMS. Meteorol. Atmos. Phys., 49: 69-91, 1992.

RAMOS DA SILVA, R.; V. P. SILVA FILHO; A. W. GANDU; P. SATYAMURTY; M. I. VITORINO; A. A. COSTA. Tunning RAMS to Northeast Region of Brazil - Preliminary Results. Seventh Conference on Mesoscale Processes., Reading, UK, American Meteorological Society, 435-436, 1996a.

RAMOS DA SILVA, R.; M. I. VITORINO; A. A. COSTA; K. C. MENDES; A. C. C. LUZ; M. SAKAMOTO; V. P. SILVA FILHO; A. W. GANDU. A experiência da FUNCEME na Utilização do modelo RAMS como Ferramenta de Previsão Numérica de Tempo. Anais do IX Congresso Brasileiro de Meteorologia, Campos do Jordão, SP, SBMET, 1996b.

RAMOS DA SILVA, R.; R. AVISSAR; M. A. SILVA DIAS; P. L. SILVA DIAS. A Large Eddy Simulation (LES) of the Boundary Layer Evolution Over a Deforested Region of Rondonia. 2nd International LBA Scientific Conference, Manaus, AM, 2002.

RAMOS DA SILVA, R.; R. AVISSAR. Large eddy simulations (LES) of wet convection in Rondonia (Amazon). International Union of Geophysics and Geodesy - IUGG, Saporo, Japan, IUGG, 2003.
RAMOS DA SILVA, R.; P. L. SILVA DIAS; A. W. GANDU; D. S. MOREIRA. Impactos da Temperatura da Superficie do Mar no Cliclone Catarina. Congresso Brasileiro de Meteorologia, Fortaleza-CE, SBMET, 2004.

RAMOS DA SILVA, R. Estudo Numérico com o Modelo OLAM para a America do Sul. Anais do XIV Congresso Brasileiro de Meteorologia, SBMET, Florianópolis-SC, 2006.

RAMOS DA SILVA, R.; P. L. SILVA DIAS, D. S. MOREIRA; V. H. N.A. LINS. Analise da performance do modelo OLAM na previsão climática para a América do Sul. Anais da III Conferencia Regional sobre Mudanças Globais: América do Sul, São Paulo-SP, 2007.

RAMOS DA SILVA, R.; D. WERTH; R. AVISSAR. Regional Impacts of Future Land-Cover Changes on the Amazon Basin Wet-Season Climate. J. Climate, 21 (6), 1153-1170, 2008.

RAMOS DA SILVA, R.; R. AVISSAR. The hydrometeorology of a deforested region of the Amazon. J. Hydrometeorol., (7), 1028-1042, 2006.

ROBERTSON, A. W.; C. MECHOSO. Interannual and decadal cycles in river flows of southeastern South America. J. Climate. 11, 2570-2581, 1998.

ROPELEWSKI, C. F.; M. S. HALPERT. Global and regional scale precipitation patterns associated with the El-Nino Southern Oscillation. Mon. Wea. Rev., 115, 1606-1626, 1987.

SELUCHI, M. E.; J. A. MARENGO. Tropical-midlatitude exchange of air masses during summer and winter in South America: Climatic aspects and examples of intense events. Int. J. Climatol., 20, 1167-1190, 2000.

SILVA DIAS, M. A., e COAUTHORS. River breeze circulation in eastern Amazonia: observations and modelling results. Theor. Appl. Climatol.,78, 111-121, 2004.

SILVA DIAS, M. A. e COAUTHORS. A case study of convective organization into precipitating lines in the Southwest Amazon during the WETAMC and TRMM-LBA. J. Geophys. Res., 107, doi:10.129/2001JD000375, 2002 a.

- Cloud and rain processes in a biosphere-atmosphere interaction context in the Amazon Region. J. Geophys. Res., doi:10.129/2001JD000335, $2002 b$.

SILVA, V.B. S.; V. E. KOUSKY; W. SHI; R. W. HIGGINS. An Improved Gridded Historical Daily Precipitation Analysis for Brazil. J. Hydrometeor., 9, 847-861, 2007.

SOLOMON, S. e CO-AUTHORES. IPCC-Report. Climate Change. The Scientific Basis. Cambridge University Press., 2007.

SOUZA, E. B.; M.T. KAYANO; J. TOTA; L. PEZZI; G. FISCH; C. NOBRE. On the influences of the El Niño, La Niña and Atlantic dipole pattern on the Amazonian rainfall during 1960-1998. Acta Amazonica, 30(2): 305-318, 2000. 
SOUZA, E. B.; T. AMBRIZZI. ENSO impacts on the South American rainfall during 1980s: Hadley and Walker circulation. Atmósfera, 15: 105-120, 2002.

SOUZA, E. B.; AMBRIZZI, T.; COELHO, C. A. S. Two ENSO episodes with reversed effects on the regional precipitation of the northeastern South America. Meteorologica, Buenos Aires - Argentina, v 29, n. 1, p. 5-16, 2004.

SOUZA, E. B.; M. T. KAYANO; T. AMBRIZZI. Intraseasonal and submonthly variability over the eastern Amazon and Northeast Brazil during the autumn rainy season. Theor. App. Climatol., 81: 177-191, 2005.

SOUZA, E. B.; AMBRIZZI, T. Modulation of the intraseasonal rainfall over tropical Brazil by the Madden-Julian Oscillation. Int. J. Climatol., v. 26, p. 1759-1776, 2006.

STROEVE, J. C.; M. C. SERREZE; F. FETTERER; T. ARBETTER; W. MEIER; J. MASLANIK; K. KNOWLES. Tracking the Artic's shrinking ice cover: Another extreme September minimum in 2004. Geophys. Res. Lett., 32, L04501, doi:10.129/2004GL021810, 2005.

TREMBACK, C. J. Numerical simulation of a mesoscale convective complex: model development and numerical results. PhD. Dissertation, Atmos. Sci. Paper 465, Colorado
State University, Dept. of Atmospheric Sciences, Fort Collins, CO, 1990.

WALKO, R. L.; W. R. COTTON; M. P. MEYERS; J. Y. HARRINGTON. New RAMS cloud microphysics parameterization .1. The single-moment scheme. Atmos. Res., 38, 29-62, 1995.

WALKO, R. L.; R. AVISSAR. The Ocean-Land-Atmosphere Model (OLAM). Part I: Shallow-water testes. Mon. Wea. Rev., 136, 4033-4044, 2008.

WALKO, R. L.; COAUTHORS, 2000: Coupled atmospherebiophysics-hydrology models for environmental modeling. J. Appl. Meteor., 39, 931-944.

WANG, H.; R. FU. Influence of cross-Andes flow on the South American low-level jet. J. Climate. 17, 1247-1262, 2004.

WEBSTER, P. J.; G. J. HOLLAND; J. A. CURRY; H.-R. CHANG. Changes in tropical cyclone number, duration, and intensity in a warming environment. Science. 309, 1844-1846, 2005.

WENNEKER, I.; A. SEGAL; P. WESSELING. A Mach-uniform unstructured staggered grid method. Int. J. Numer. Meth. Fluids, 40, 1209-1235, DOI: 10.1002/fld.417, 2002. 